\title{
Unusual Presentation of Neurocysticercosis: Report of Two Cases
}

\author{
Ghanashyam Gurung, MD \\ Department of Radiology and Imaging \\ Tribhuvan University Teaching Hospital \\ Kathmandu \\ Nepal
}

\author{
Bhiva Shrestha, MD \\ Department of Radiology and Imaging \\ Tribhuvan University Teaching Hospital \\ Kathmandu \\ Nepal
}

\section{Tilak C. Shah, DMRD}

Department of General Practice

Tribhuvan University Teaching Hospital Kathmandu

Nepal

\section{Laxman R. Pokhrel, MBBS}

Department of Radiology and Imaging Tribhuvan University Teaching Hospital Kathmandu

Nepal

\section{Geeta Sayami, MD, PhD}

Department of Pathology

Tribhuvan University Teaching Hospital Kathmandu

Nepal

\author{
Sushil K. Shilpakar, MS \\ Division of Neurosurgery \\ Tribhuvan University Teaching Hospital \\ Kathmandu \\ Nepal
}

\author{
Address for correspondence: \\ Ghanashyam Gurung, MD \\ Department of Radiology and Imaging \\ Tribhuvan University Teaching Hospital \\ Kathmandu \\ Nepal \\ Email: puspaghan@mos.com.np
}

Received, Novemebr 3, 2004

Accepted, Decemebr 23, 2004

$\mathrm{N}$ eurocysticercosis is the most common parasitic infestation of the central nervous system caused by larvae (cysticerci) of pork tapeworm (Taenia solium). Millions of people residing in developing nations of Central and South America, Sub Saharan Africa, South and East Asia have been the sufferers of this disease. 1,2,7,15 Globally, more than 50,000 new NCC related deaths occur
Neurocysticercosis (NCC) is the most common parasitic infestation of the central nervous system, and is one of the major public health problems in developing nations like Nepal. On imaging studies, NCC usually demonstrates multiple parenchymal ring enhancing lesions in the gray white matter interface of the brain, though presence of nonenhancing vesicles with scolex or calcified dots is also a frequent finding. Co-existence of brain abscess and neurocysticercosis is a rarity. Presentation of NCC in the form of a large cystic mass in the $4^{\text {th }}$ ventricle is also unusual. Here, we present these unusual cases of neurocysticercosis, which remained in their masks until they were surgically explored and microscopically unveiled!

Key Words: brain abscess, CT, MRI, Neurocysticercosis per annum. Young people usually tend to present with the most awful neurological manifestations and sequele. ${ }^{6,17} \mathrm{NCC}$ has undoubtedly been one of the major public health problems in developing nations that has imparted a profound impact upon health, productivity and quality of life. Newer neuro-imaging modalities, such as computed tomography (CT) and magnetic resonance imaging (MRI) scanning, have improved the diagnostic accuracy of this disease. However, in some instances diagnosis is not reached until examined histopathologically. We came across two patients whose initial presentation was not typical of NCC. We describe these two cases in detail along with a brief of the literature.

\section{Case Reports}

\section{Case 1}

History

This 21-year-old-lady presented to us with a 2-month history of progressive headache and diminution of vision.

\section{Examination}

General physical examination revealed no abnormality. Neurological evaluation revealed no abnormal findings except for bilateral papilledema. MRI of the brain revealed a well-defined cystic mass with eccentric mural nodule in the fourth ventricle with mild to moderate obstructive hydrocephalus. The cyst appeared hypointense in T1WI and hyperintense in T2WI. No enhancement was noted in postcontrast study (Figure 1A and 1B). MRI diagnosis was a parasitic cystic lesion, most likely neurocysticercosis.

\section{Hospital course}

Routine hematological and biochemical parameters were within normal limits. The patient underwent placement of a ventriculo-peritoneal shunt followed by a suboccipital craniectomy. At operation the cyst was easily peeled off the 


\section{Neurocysticercosis}
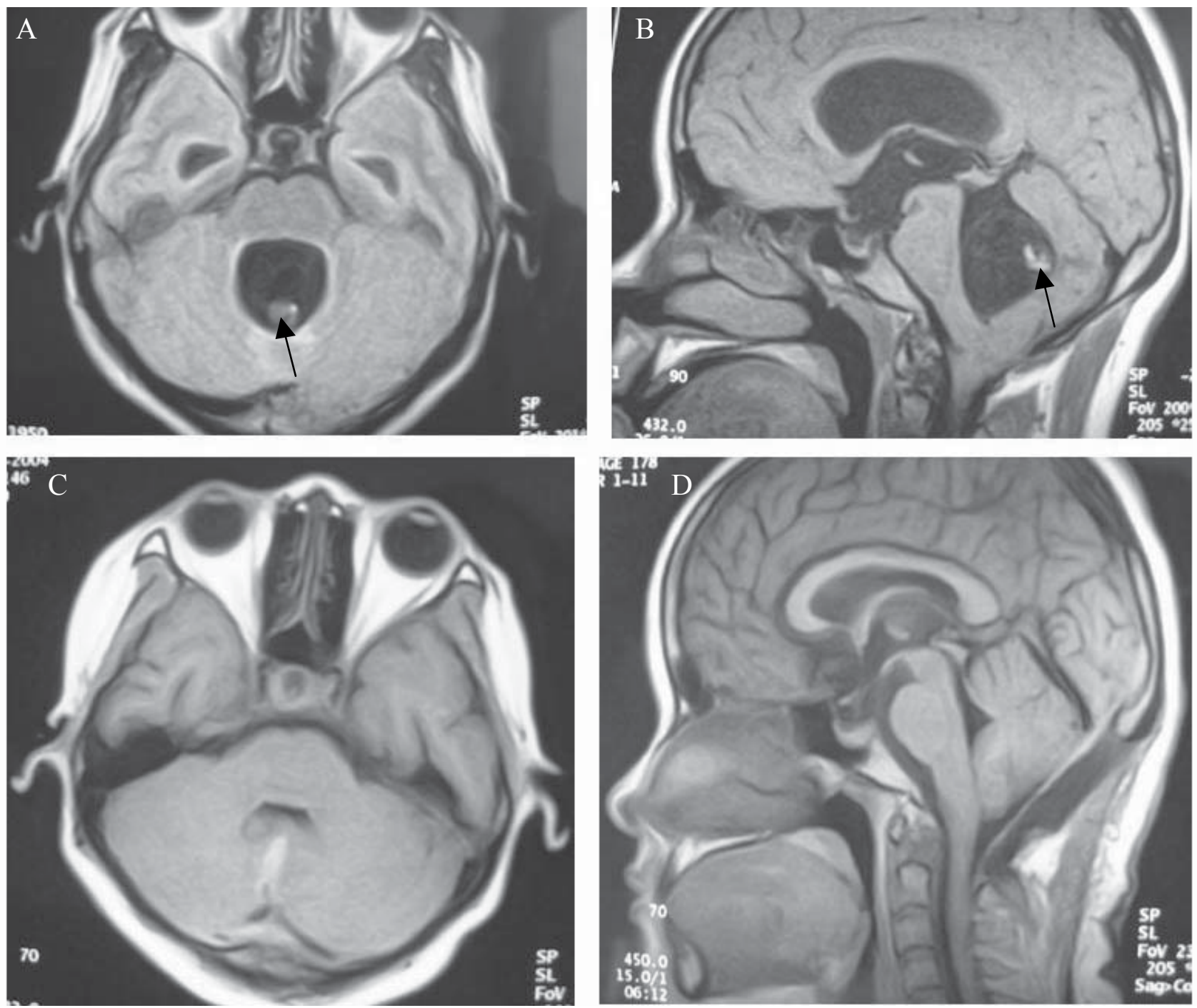

Figure 1. MRI head A) TIWI axial and B)TIWI sagittal of Case 1 showing a well-defined cystic lesion with a mural nodule (arrow). C\&D: Post-operative MRI showing complete excision of the lesion.

adjacent brain tissue and total removal was achieved.

The patient tolerated the surgery well. Histopathologically, the sections showed a bladder wall with tegumental surface having protuberances and beneath it there were small pyknotic nuclei and loose connective tissue (Figure 2). Thus the histopathological diagnosis of neurocysticercosis was established. An MRI obtained 2 weeks later showed no evidence of the lesion (Fig 1C and 1D). The patient was discharged home in 3 weeks in stable condition.

\section{Case 2}

\section{History}

This 52-year-old gentleman presented with slurring of speech and deviation of the mouth towards the left side for one month and weakness of the right half of the body for one week. There was no history of fever, vomiting or loss of consciousness. The patient was known to have been hypertensive for eight years and diabetic for four years, on regular medication.

\section{Examination}

General physical examination revealed no abnormality. On neurological examination he had right 7 th cranial nerve paresis and motor dysphasia. The reminder of his examination was normal. CT scan of his head revealed a solitary, well defined, approximately $3 \times 4 \mathrm{~cm}$ sized, intensely enhancing ring-like lesion in the left parietal lobe with a smooth and regular wall (Figure 3A and 3B). Minimal perilesional edema was noted but there was no significant mass effect. Enhancing intramural nodule or calcification within the lesion was absent. Considering CT morphological characteristics, a diagnosis of left parietal lobe abscess was put forth.

\section{Hospital course}

The patient underwent burrhole and drainage of the abscess. At surgery thick yellow pus was obtained, which on subsequent culture grew Staphylococcus aureous. Small flecks of debris were sent for histopathological examination. Histopathology revealed cysticercosis (Figure 4). In addition, the patient received antibiotics and an anticon- 

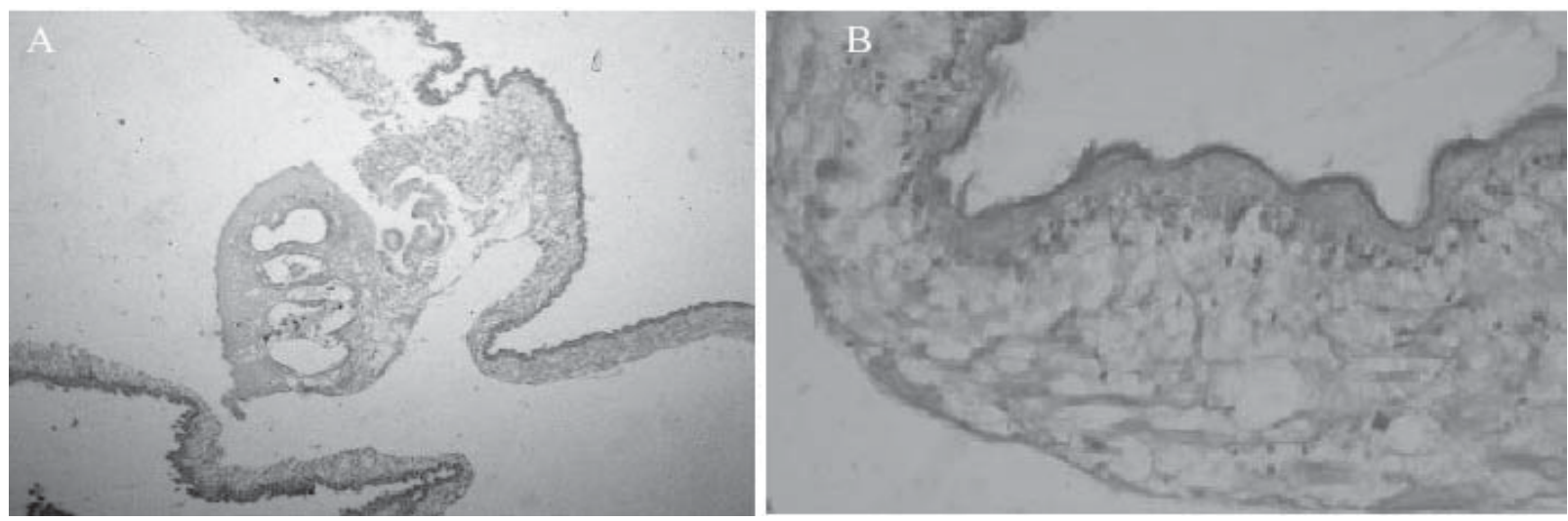

Figure 2. Photomicrographs of tissue specimen of Case 1. The section shows a bladder wall with tegumental surface having protuberances and beneath it, there are small pyknotic nuclei and loose connective tissue. Histopathological diagnosis is cysticercosis. ( $H \& E$ stain, $A(x 40) ; B(x 200)$ ).

vulsant. A postoperative follow up CT scan showed reduction in the size of the lesion. Additionally, a small eccentric mural nodule was visualized confirming the histopathologic findings (Fig3C).

\section{Discussion}

Neurological manifestations of cysticercosis vary and are related to the size, number and location of the parasites. Epilepsy is the most common clinical manifestation occurring in almost $70 \%$ of patients with $\mathrm{NCC}^{5}$ and is most commonly generalized tonic-clonic in type. ${ }^{5,13}$ There was no history of seizure in both of our cases. Cysticercal arachnoiditis, granular ependymitis or presence of intraventricular cysts may lead to hydrocephalus, increased intracranial pressure and dementia. ${ }^{8}$ Increased intracranial pressure (ICP) also occurs in patients with giant cysts. In our first case, the patient was symptomatic of hydrocephalus due to obstruction at the $4^{\text {th }}$ ventricle.

Cysticerci are essentially vesicles with two major parts; vesicular wall and the scolex. The scolex has a similar morphology to the adult worm with a rudimentary body and an armed rostellum. ${ }^{14}$ Viable cysticerci in brain parenchyma measure approximately $10 \mathrm{~mm}$ in diameter. In more than half of the cases, brain parenchyma is affected. ${ }^{11}$ The corticomedullary junction is the most favored location in the brain. Only $10 \%$ of NCC cases have isolated subarachnoid disease. In subarchnoid spaces cysticerci may be as large as $50 \mathrm{~mm}$ or more because of their unrestrained growth unlike in the parenchyma. Sometimes, several intervening membranes may attach a cluster of cysts imparting a bunch of grape-like appearance (racemose form of cysticercosis). This is due to developmental arrest and the scolex is never formed in this variety. ${ }^{12}$ Though the scolex is not seen, the lesion can be quite large.

More than one anatomic compartment is often involved. ${ }^{3}$ Teitelbaum, et al., found that $20 \%$ to $50 \%$ of intraventricular cysticerci are located in the fourth ventricle, the commonest site among the ventricles. ${ }^{20}$ However, Khandelwal, et al., maintain that intraventricular cysticerci constitute only $7 \%$ to $25 \%$ of the total infection and have observed a maximum frequency of $33 \% .{ }^{10}$

CT and MR imaging findings in parenchymal disease depend upon the developmental stage of the cysticerci. Characteristic imaging features include the scolex appearing as a hyperintense nodule or as a punctate calcific focus ${ }^{19}$. Single or multiple ring / nodular enhancing lesions constitute the most frequent findings.

On imaging, ventricular cysticerci appear as cystic lesions that distort the anatomy of the ventricular system and cause asymmetrical hydrocephalus. ${ }^{9,18}$ The lesions are usually isodense to CSF on MR imaging but are rarely recognizable on plain CT scans. Concurrent administration of intravenous contrast medium during CT may help in visualizing them. On MR, most of the ventricular cysts are detected since the scolex is better delineated and the signal properties of the cystic fluid and CSF are different on T2WI. $9,11,18$ In our first case, the cyst occupied the whole of the fourth ventricular surface, which showed an eccentrically located hyperintense nodule, the scolex. This lesion completely obstructed the CSF flow tract leading to hydrocephalus.

Although parenchymal in location, there were no classic features of cysticercosis in our second case and it co-existed with a bacterial abscess. No scolex could be seen on initial CT scan. Parenchymal neurocysticercosis is sometimes difficult to differentiate from pyogenic brain abscess, fungal abscess, tuberculoma, toxoplasma abscess and some brain tumors which have similar ring enhancement characteristics to NCC. ${ }^{1,4,16,17}$ Co-existence of brain abscess and NCC has not been previously reported in the literature. In our case, the lesion was large showing a smooth enhancing wall with perilesional edema favoring the diagnosis of abscess rather than neurocysticercosis as the initial diagnosis. This unusual presentation may be due to secondary infection of the cyst or mere coexistence as a chance phenomenon.

Parenchymal calcifications usually remain the sole evidence of the disease in many patients. CT remains the best neuroimaging screening modality in patients with suspected NCC as small parenchymal calcifications are better appreciated on CT than on MRI. MRI may be reserved 

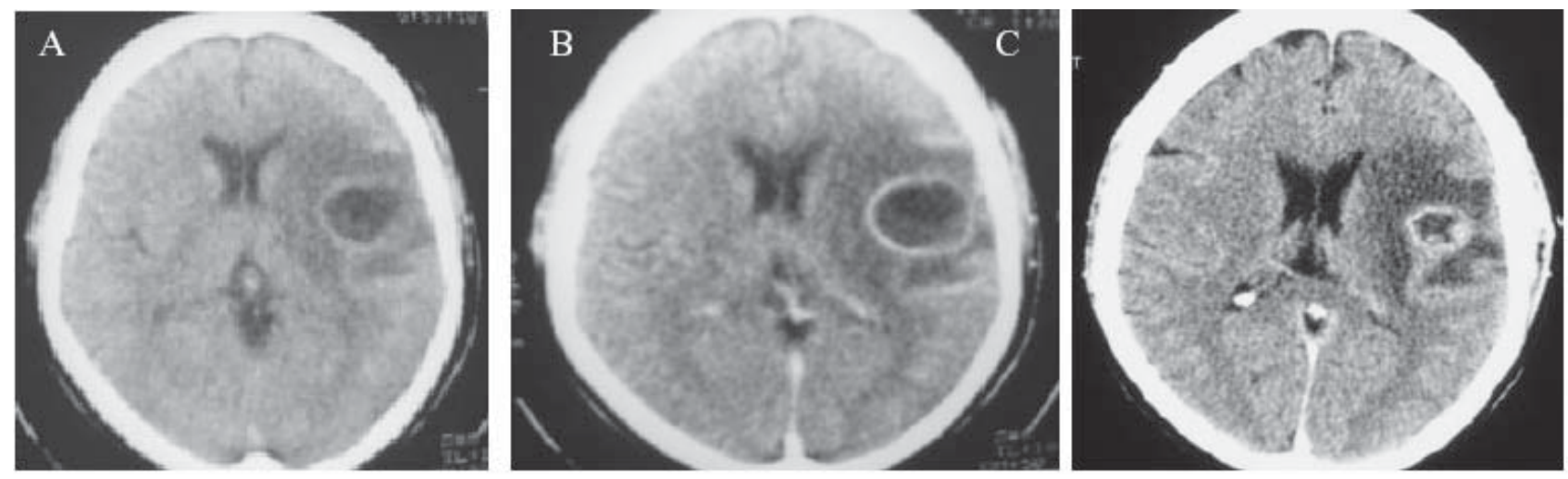

Figure 3. CT scan of case 2, A) noncontrast and contrast enhanced CT scan showing a ring enhancing lesion with surrounding edema. Post operative contrast enhanced scan $(C)$ showing significant reduction in the size of the lesion.

for patients in whom CT scan doesn't demonstrate evidence of these lesions and in situations when CT findings are inconclusive $^{18}$. In general, MRI is better than CT in the diagnosis of NCC, particularly with the skull base, brainstem, intraventricular or spinal lesions.

\section{Conclusions}

NCC may pose a great diagnostic challenge in imaging when it presents with atypical morphology, though the majority of the lesions are virtually quite diagnostic. Possibility of its presence should always be borne in mind if a ring enhancing lesion is discovered. With a cystic lesion

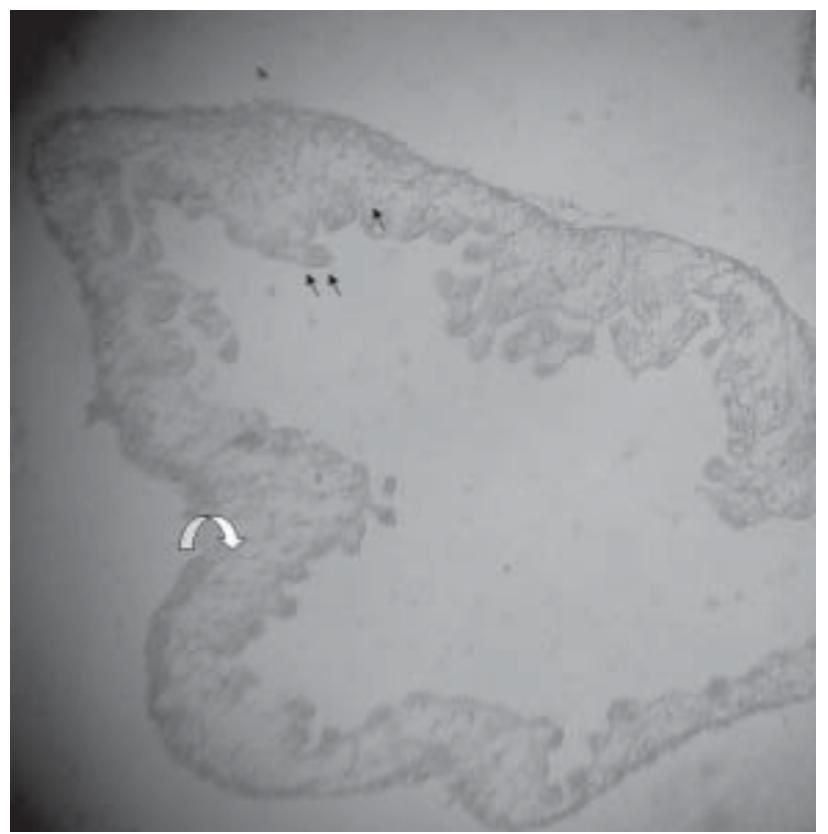

Figure 4. The bladder wall of a cysticercus shows three distinct layers. The tegumental surface has wart-like protuberances (double arrows) and is acidophilic. Directly beneath the tegument are small, rounded, pyknotic nuclei (arrow). The innermost layer is made up of loose connective tissue (curved arrow). This is diagnostic of cysticercosis. ( $H \&$ E stain , (x10) especially in the fourth ventricle, diagnosis of NCC should always be considered.

\section{Acknowledgments}

We thank Abhimanyu Jha, MD from the Department of Pathology, TU Teaching Hospital for helping in the preparation of the manuscript.

\section{References}

1. Chandy MJ, Rajshekhar V, Ghosh S, et al: Single small enhancing CT lesions in Indian patients with epilepsy: clinical radiological and pathological considerations. J Neurol NeurosurgPsychiat 54:702-705, 1991

2. Coker-Vann MR, Subianto DB, Brown P, et al: ELISA antibodiesto cysticerci of Taenia solium in human populations in New Guinea, Oceania, and Southeast Asia. Southeast Asian J Trop Med Public Health 12:499-505, 1981

3. Couldwell WT, Zee C-S, Apuzzo MLJ: Definition of the role of contemporary surgical management in cisternal and parenchymatous cysticercosis cerebri. Neurosurgery 28:231- 237,1991

4. Del Brutto $\mathrm{OH}$ : Single parenchymal brain cysticercus in the acute encephalitic phase: definition of a distinct form of neurocysticercosiswith benign prognosis. J Neurol Neurosurg Psychiat 58:247249, 1995

5. Del Brutto OH, Santibañez R, Noboa CA, et al: Epilepsy due to neurocysticercosis: analysis of 203 patients. Neurology 42:389-392, 1992

6. Del Brutto $\mathrm{OH}$, Sotelo J, Román GC: Neurocysticercosis: A Clinical Handbook. Lisse, The Netherlands: Swets \& Zeitlinger, 1998

7. Díaz F, García HH, Gilman RH, et al: Epidemiology of taeniasisand cysticercosis in a Peruvian village. The Cysticercosis Working Group in Peru. Am J Epidemiol 135:875-882, 1992

8. Estañol B, Kleriga E, Loyo M, et al: Mechanisms of 
hydrocephalus in cerebral cysticercosis: implications for therapy. Neurosurgery 13:119-123, 1983

9. Ginier BL, Poirier VC: MR imaging of intraventricular cysticercosis. AJNR Am J Neuroradiol 13:1247-1248, 1992

10. Khandelwal S., Sakhi P., Sharma GL, et al: Intraventricullar cysticercos ind. J Radiol Image 12:329-332, 2002

11. Martinez HR, Rangel-Guerra R, Elizondo G, et al: MR imaging in neurocysticercosis: a study of 56 cases. AJNR Am J Neuroradiol 10:1011-1019, 1989

12. Martinz A., Anatomia pathlogicadelacisticercosis cerebral . Neurocirugia 19:191-201, 1961

13. Medina MT, Rosas E, Rubio-Donnadieu F, et al: Neurocysticercosis as the main cause of late-onset epilepsy in Mexico. Arch Intern Med 150:325 327,1990

14. Pittella JEH: Neurocysticercosis. Brain Pathol 7:681-693, 1997
15. Preux PM, Melaku Z, Druet-Cabanac M, et al: Cysticercosis and neurocysticercosis in Africa: current status. Neurol Infect Epidemiol 1:63-68, 1996

16. Rangel R, Torres B, Del Bruto O, et al: Cysticercotic encephalitis:a severe form in young females. Am J Trop Med Hyg 36:387-392, 1987

17. Roman G, Sotelo J, Del Brutto O, et al: A proposal to declare neurocysticercosis an international reportable disease. Bull World Health Organ 78:399-406, 2000

18. Salgado P, Rojas R, Sotelo J: Cysticercosis. Clinical classification based on imaging studies. Arch Intern Med 157:1991-1997, 1997

19. Suss RA, Maravilla KR, Thompson J: MR imaging of intracranial cysticercosis: comparison with CT and anatomopathologic features. AJNR Am J Neuroradiol 7:235-242, 1986

20. Teitelbaum GP, otto RJ, Lin M et.al :MR imaging of neurocysticercosis: AJNR Am J Neuroradiol 10:709-718, 1989 